Check for updates

Cite this: RSC Adv., 2017, 7, 22619

\title{
From wheat bran derived carbonaceous materials to a highly stretchable and durable strain sensor $\uparrow$
}

\begin{abstract}
Jing Ren, ${ }^{a}$ Xuan Du, ${ }^{a}$ Wenjun Zhang (iD ${ }^{a}$ and Ming Xu (D) *ab
Highly stretchable strain sensors with exceptional sensitivity and durability are essential for applications in human health monitoring, soft robotics, etc. The majority of stretchable strain sensors are composite materials using conductive fillers to produce sensitivity and an elastic polymer to acquire stretchability. However, it is difficult to develop highly sensing strain sensors meanwhile possessing stretchability, durability and frequency stability, all of which are particularly important for real use. The high strains applied always cause stress concentration around the conductive network, leading to unrepaired cracks during cyclic operations. It is challenging to design an appropriate composite structure where the interaction between fillers and polymers remains stable upon large deformations with minimal stress concentration. In this work, we created a strain sensor with a conductive network stacked with highly homogeneous carbon fragments from wheat bran derived carbonaceous materials, each of which apportioned the strain as equally as possible to minimize the local deformation and stress concentration. The sensor showed excellent durability over 10000 stretching cycles at $80 \%$ strain with high sensitivity (gauge factor of 62.8) and frequency stability $(0.01-1 \mathrm{~Hz}$ ), among the highest recorded for stretchable strain sensors based on nanocarbon materials and biomass materials.
\end{abstract}

Received 14th February 2017

Accepted 17th April 2017

DOI: $10.1039 / \mathrm{c} 7 \mathrm{ra01837a}$

rsc.li/rsc-advances aggregation of nanomaterials during mixture or curing process dramatically worsens the interaction with the matrix, meanwhile resulting in the stress concentration at interfaces. ${ }^{23}$ As is known, stress concentration, the origin of cracking, is a dominant factor that cause fatigue fracture of materials, which is needed to be restrained through structural design or facile fabrication. ${ }^{24}$ Although the chemical modification (e.g. oxidation treatment and adding surfactant) can effectively suppress the aggregation, ${ }^{25,26}$ it would downgrade the conductivity of the electrical pathways, therefore making sensors unstable and unmeasurable. As seen, it is challenging to make filler materials uniformly distributed to minimize stress concentration meanwhile maintaining their conductivity, which actually is the foundation for achieving the combination of high sensitivity, stretchability and durability for strain sensors.

Biomass materials, particularly the cellulose-rich species have become promising candidates for stretchable strain sensors. Through simple thermal treatments, the cellulose network in biomass materials can be transformed into the highly conductive pathways to provide electrical signals upon stretching. Tissue paper was tried in the stretchable strain sensor, showing the high gauge factor of 25.3 within the $3 \%$ tensile strain..$^{27}$ However, the long-term operation was not able to be conducted at the strain beyond $3 \%$, due to the irreversible damage of the tissue-derived conductive pathway. Recently, a cotton fabric has been used as recoverable conductive network for a stretchable strain sensor workable at $50 \%$ strain up to 2000 cycles with a very high gauge factor $(25-64) .{ }^{28}$ When combining
${ }^{a}$ State Key Laboratory of Materials Processing and Die \& Mould Technology, School of Materials Science and Engineering, Huazhong University of Science and Technology, Wuhan, 430074, China. E-mail: ming.xu@hust.edu.cn

${ }^{b}$ Shenzhen R\&D Center of Huazhong University of Science and Technology, Shenzhen 518000, China

$\dagger$ Electronic supplementary information (ESI) available. See DOI: 10.1039/c7ra01837a 
with the multi-directional weaving design of silk fabric, the durability of the strain sensor was promoted to 6000 cycles at a remarkably high strain of $100 \% .^{29}$ As can be seen, all the biomass materials by far chosen for the stretchable strain sensors have a common in their interconnected fabric structure, which was applied as the conductive network perform in sensors without exception. It provided an efficient route to avoid the aggregation of filler materials during fabrication process, giving rise to an improvement in durability of sensors. However, such methodology restricts the promotion/ optimization of sensing performances in further steps, since their performance and working mechanism as well were established based on the original configuration of source materials rather than preparation processing. ${ }^{27-29}$

In this study, we reported a highly stretchable and durable strain sensor using wheat bran as a source material. Different from the specific micro-patterns of biomass materials being directly used as conductive network perform in strain sensors, our approach to achieve the superior durability at high strains was to assemble the carbon fragments made from wheat bran derived carbonaceous materials through drop casting, in which the sensing performance was being optimized by tuning the concentration of carbon fragments. The efforts were focused to prepare the carbon fragments in an exceptionally uniform size distribution, aiming to create a conductive network stacked with highly homogeneous units, each of which was capable to apportion the strain as equally as possible to minimize the local deformation and stress concentration around. Such structural design was demonstrated to provide our sensor with an excellent durability over 10000 stretching cycles at the tensile strain up to $80 \%$, which is among the highest records of the stretchable strain sensors based on the nanocarbon materials and biomass materials (see Table S1 $\dagger$ ). Moreover, it showed high sensitivity (gauge factor of 62.8) and frequency stability (0.01-1 $\mathrm{Hz}$ ) at large strains that was capable of monitoring various human motions such as the bending of elbow, walking, dancing and running.

\section{Experimental}

\section{Preparation of carbon fragments}

Firstly, 1 gram wheat bran powder and $40 \mathrm{~mL}$ distilled water were added into a $50 \mathrm{~mL}$ Teflon-lined stainless autoclave. Hydrothermal reaction was conducted at $180{ }^{\circ} \mathrm{C}$ for $12 \mathrm{~h}$ and then cooled to room temperature naturally. The obtained samples were frozen completely by liquid nitrogen and freezedried for $24 \mathrm{~h}$. The hydrothermal treated wheat bran and potassium hydroxide $(\mathrm{KOH})$ were thoroughly ground in an agate mortar at the weight ratio of $1: 2$, and then the mixture was heated at $800{ }^{\circ} \mathrm{C}$ for $2 \mathrm{~h}$ under argon flow. After that, the activated sample was thoroughly washed with dilute hydrochloric acid $(\mathrm{HCl})$ and then distilled water until the $\mathrm{pH}$ became neutral, and dried at $100{ }^{\circ} \mathrm{C}$ for $12 \mathrm{~h}$. The collected samples were named as carbon fragments. The carbon fragments was dispersed in isopropyl alcohol (IPA) at the different concentration.

For comparison, the graphene oxide (GO) was prepared according to the modified Hummers' method. ${ }^{30}$ Then, the GO dispersion at the concentrations of $1 \mathrm{mg} \mathrm{mL} \mathrm{mL}^{-1}$ was sonicated using a ultrasonic bath cleaner $(150 \mathrm{~W})$ until it became clear with no visible particulate matter. Then, the GO was reduced by hydrazine hydrate. Hydrazine hydrate $(1 \mathrm{~mL})$ was then added and the dispersion heated in an oil bath at $100{ }^{\circ} \mathrm{C}$ under a watercooled condenser for 24 h over which the reduced GO gradually precipitated out as a black solid. This product was washed with water and ethanol, and dried to obtain the reduced graphene oxide (rGO). ${ }^{31}$

\section{Preparation of CFs/PDMS strain sensor}

A pattern with a size of $58 \mathrm{~mm} \times 10 \mathrm{~mm}$ was formed on a glass slide $(76 \mathrm{~mm} \times 26 \mathrm{~mm}$ ) by using polyimide tape. The $1 \mathrm{~mL}$ of CFs/IPA dispersion was drop-casted onto the patterned glass slide with a micropipette. The sample was dried at $70{ }^{\circ} \mathrm{C}$ for $4 \mathrm{~h}$ to remove the IPA. After the removal of the polyimide tape, the liquid polydimethylsiloxane (PDMS) pre-elastomer, a viscous mixture of base/curing agent (10:1, wt/wt) (Sylgard 184, Dow Corning, USA) was poured onto the glass slide and it was cured at $70{ }^{\circ} \mathrm{C}$ for $2 \mathrm{~h}$. Then, the CFs/PDMS composite film was peeled off from the glass slide and copper wires were connected to film with silver paste for electrical characterization. The rGO/PDMS strain sensor was prepared using the same drop-casting procedure.

\section{Characterization}

The morphology was observed with field emission scanning electron microscope (FE-SEM) (Nova Nano SEM 450). Transmission electron microscopy (TEM) observation was carried out on FEI Tecnai G2 F20 S-TWIN. Optical images were taken by a Nikon ECLIPSE LV150 microscope with a Nikon Digital Sight DS-Fi1 camera. The structure was checked by X-ray diffraction (XRD, PANalytical B.V.) and Raman spectra (Bruker VERTEX 70). The sheet resistance was measured by Mitsubishi four-point conductivity probe by using a linear arrayed four-point head (MCP-T370). To test the sensing properties, the stretching/ releasing tests were carried out on the Shimadzu Universal Testing Machine (AGS-5KNX) and the electrical resistance changes were recorded by a digital source meter (Agilent 34461A Digital Multimeters). The current-voltage curves were recorded by the $\mathrm{CHI}$ 660C electrochemical workstation ( $\mathrm{CH}$ Instruments Inc.).

\section{Results and discussion}

The fabrication process of the carbon fragments (CFs) and strain sensor was illustrated in Fig. 1a. Wheat bran was carefully chosen for its natural configuration of fine grained chippings with the size of 1-3 mm, which was easier to be downsized from macroscopic chipping to microscopic fragment. During the hydrothermal carbonization process, the water molecule infiltrated into the cell walls of wheat bran to produce amounts of 10-30 $\mu \mathrm{m}$ sized carbon pieces (Fig. 1b). Compared to the hightemperature carbonization $\left(\sim 600{ }^{\circ} \mathrm{C}\right)$ in previous reports, ${ }^{32-34}$ our hydrothermal carbonization process $\left(180^{\circ} \mathrm{C}\right)$ inhibited the coalescence of cell walls and provided adequate mixing with $\mathrm{KOH}$ powder for the following activation, thus favouring finer 


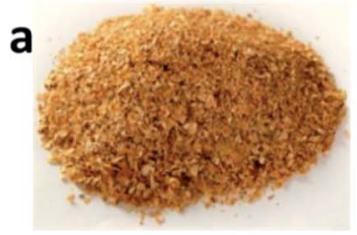

Wheat bran

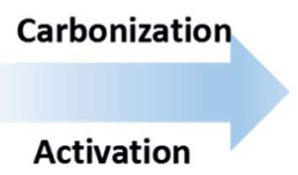

Activation

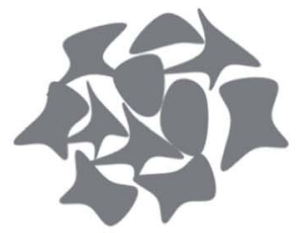

Carbon Fragments (CFs)
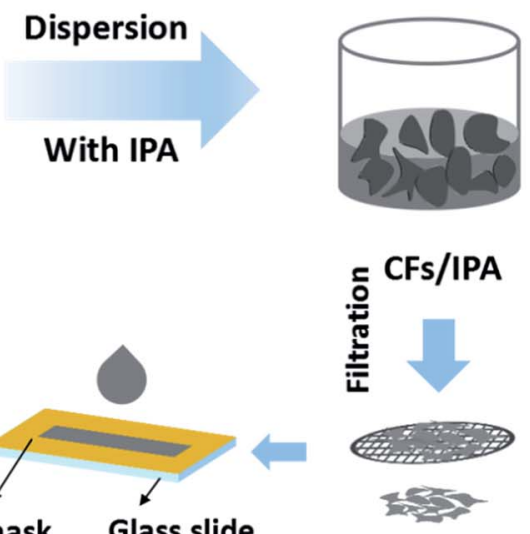
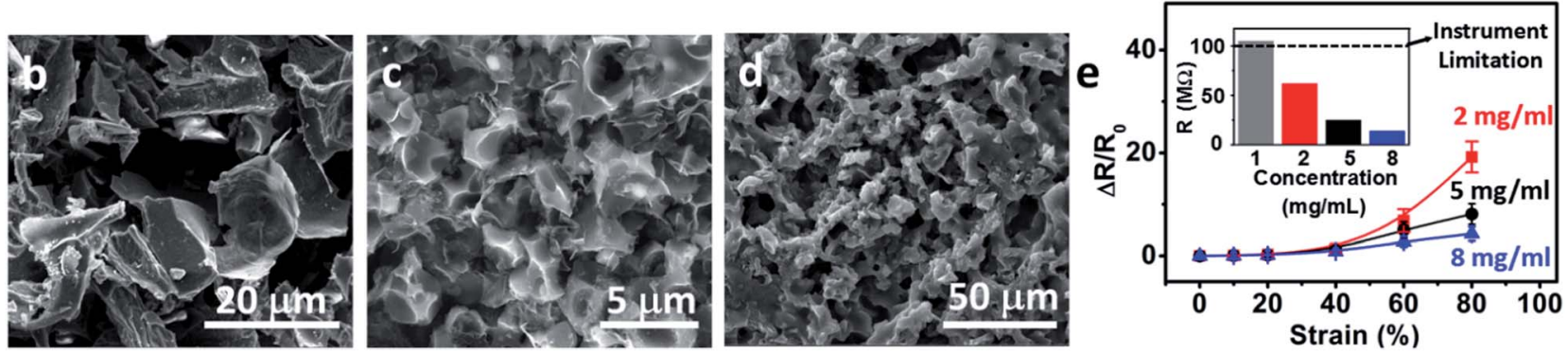

Fig. 1 (a) Schematic illustration of the fabrication process of the carbon fragments/PDMS strain sensor. (b) SEM image of carbonized wheat bran. (c) SEM image of rearranged carbon fragments after drop-casting. (d) The top view of the strain sensor where the self-contacting network structure can be maintained in the composite. (e) The relative resistance change versus strain curves of the sensor with different concentration of carbon fragments.

fragmentation (see Fig. S1†). Elemental analysis from XPS measurements revealed that the content of nitrogen (N), oxygen (O) and carbon (C) were 4.74 at\% and 12.69 at\% and 82.57 at $\%$ (see Fig. S2 and S3†). The nitrogen self-doping contributed to the improvement of electrical conductivity of the carbon pieces, while the hydrophilic oxygen functional groups improved their uniform dispersion in polar solvent. Next, these 10-30 $\mu \mathrm{m}$ carbon pieces were corroded in $\mathrm{KOH}$ (at $800{ }^{\circ} \mathrm{C}$ for 2 hours in argon atmosphere) to form a highly graphitized porous structure (see Fig. S4 $\dagger$ ) and then fragmentized by sonication in isopropyl alcohol (IPA) to be further downsized. Through the multi-level filtering by using 200, 460 and 900 mesh filter screens, we finally obtained the $5-10 \mu \mathrm{m}$ sized carbon fragments (CFs) dispersion with a highly uniform size distribution. The CFs/IPA dispersion was then drop-casted onto the open area of polyimide mask and put into oven for drying. A selfstacking conductive network was formed where every unit $\mathrm{CF}$ made contact with the adjacent ones (Fig. 1c). Upon stretching, the contacted CFs would be separated to cause the breakage of conductive pathway, representing a singularity in the resistance-strain behavior. ${ }^{35}$ In order to fabricate robust composites for stretchable strain sensor, we tried PDMS with different viscosity by tuning the weight ratio of base/curing agent for mixing, aiming to improve the adhesion between the CFs and PDMS as much as we can. Finally, the low viscous PDMS with the proper robustness (base/curing agent at the weight ratio of $10: 1$ ) was decided to be used to fabricate the samples by balancing the infiltration rate and structural robustness. On the other hand, the CFs were proceeded multi-level filtration before being mixed with PDMS matrix to minimize the geometric discontinuities, and then further increase the adhesion between two materials. A robust composite was finally fabricated without significant void under microscopic observation (Fig. 1d, S5 and S6 †).

The solution process provided the route to optimize the sensitivity by changing the concentration of CFs/IPA dispersion. The sensitivity of strain sensors, namely gauge factor (GF) was defined as $\left(\Delta R / R_{0}\right) / \varepsilon . R_{0}$ and $\Delta R$ represented the resistance under $0 \%$ strain and the resistance change at the applied strain of $\varepsilon$, respectively. We prepared our sensors with the CFs concentration ranging from $1 \mathrm{mg} \mathrm{mL}^{-1}$ to $8 \mathrm{mg} \mathrm{mL}^{-1}$ in the drop-casting procedure. The highest gauge factor of 62.8 was achieved at the applied strain of $40-80 \%$ with the CFs/IPA dispersion concentration of $2 \mathrm{mg} \mathrm{mL} \mathrm{m}^{-1}$ (Fig. 1e), while the conductive CFs film with the thickness of $22 \pm 3 \mu \mathrm{m}$ and the sheet resistance of $9.71 \pm 0.74 \mathrm{M} \Omega$ (Fig. S7 $\dagger$ ) was used. By increasing the CFs/IPA dispersion to $5 \mathrm{mg} \mathrm{mL}^{-1}$ and $8 \mathrm{mg}$ $\mathrm{mL}^{-1}$, the strain sensors exhibited higher conductivity (inset of Fig. 1e), yet lower gauge factor of 16.1 and 8.6 at the same applied strain. It was because of the denser conductive pathway formed at higher $\mathrm{CF}$ concentrations, which in fact weakened the derivative of the resistance-strain function, that is, the sensitivity of strain sensor. ${ }^{35}$ However, if the CFs concentration was decreased too much, the electrical resistance would exceed the 
limitation of instrumental measurement range $(<100 \mathrm{M} \Omega)$ to make the sensor unmeasurable. So the strain sensor made from the CFs/IPA dispersion concentration of $2 \mathrm{mg} \mathrm{mL}^{-1}$ was chosen as the optimized sample for the following tests.

The detailed electromechanical performance of our optimized strain sensors was presented in Fig. 2. The relative resistance change $\left(\Delta R / R_{0}\right)$ was used to represent the sensing property of strain sensor based on the linear current-voltage curves at different levels of strains ranged from $10 \%$ to $80 \%$ (Fig. 2a), revealing the ohmic contact behavior of our sensors. The electrical resistance increased with the increasing of applied strain, where the gauge factor was calculated from the slope of curve of the relative resistance change versus strain. As shown in Fig. 2b, the slope of curve included two relatively linear parts, $0-40 \%$ and $40-80 \%$, with a GF of 2.2 and 62.8 , respectively (Fig. S8 $\dagger$ ). In the lower strain stage of stretching (0$40 \%$ ), the change in electrical resistance was dominated by the variation of contact area between conductive carbon fragments (CFs), thereby giving rise to the low sensitivity. When beyond
$40 \%$ and entering the high strain stage, most of the contacted neighboring CFs were completely separated. The relative resistance change was dominated by the increase of the tunneling distance between separated CFs, which showed a sharp increase in the value of gauge factor. This phenomenon was also observed in other active materials-based strain sensors, such as MWCNT/PEO, ${ }^{36}$ CNT-graphene/PDMS ${ }^{37} \mathrm{Ag}$ nanowire/PDMS, ${ }^{10}$ Fragmentized graphene foam/PDMS. ${ }^{20}$ Similar as the crumpled graphene as reported, ${ }^{15}$ the three-dimensional structure of our carbon fragments was critically important for their uniform distribution in PDMS matrix by avoiding the aggregation during the drop-casting and curing process. Compared to onedimensional carbon nanotubes ${ }^{\mathbf{1 3}}$ and two-dimensional graphene, ${ }^{18}$ the contacting nodes between the neighboring CFs in our materials were significantly reduced to provide the sensitivity as high as 62.8 , which was much more sensitive than other carbon-based strain sensors. It indicated a wide operational range available for all human motions, particularly with a relatively higher sensibility for large-scale movement such as
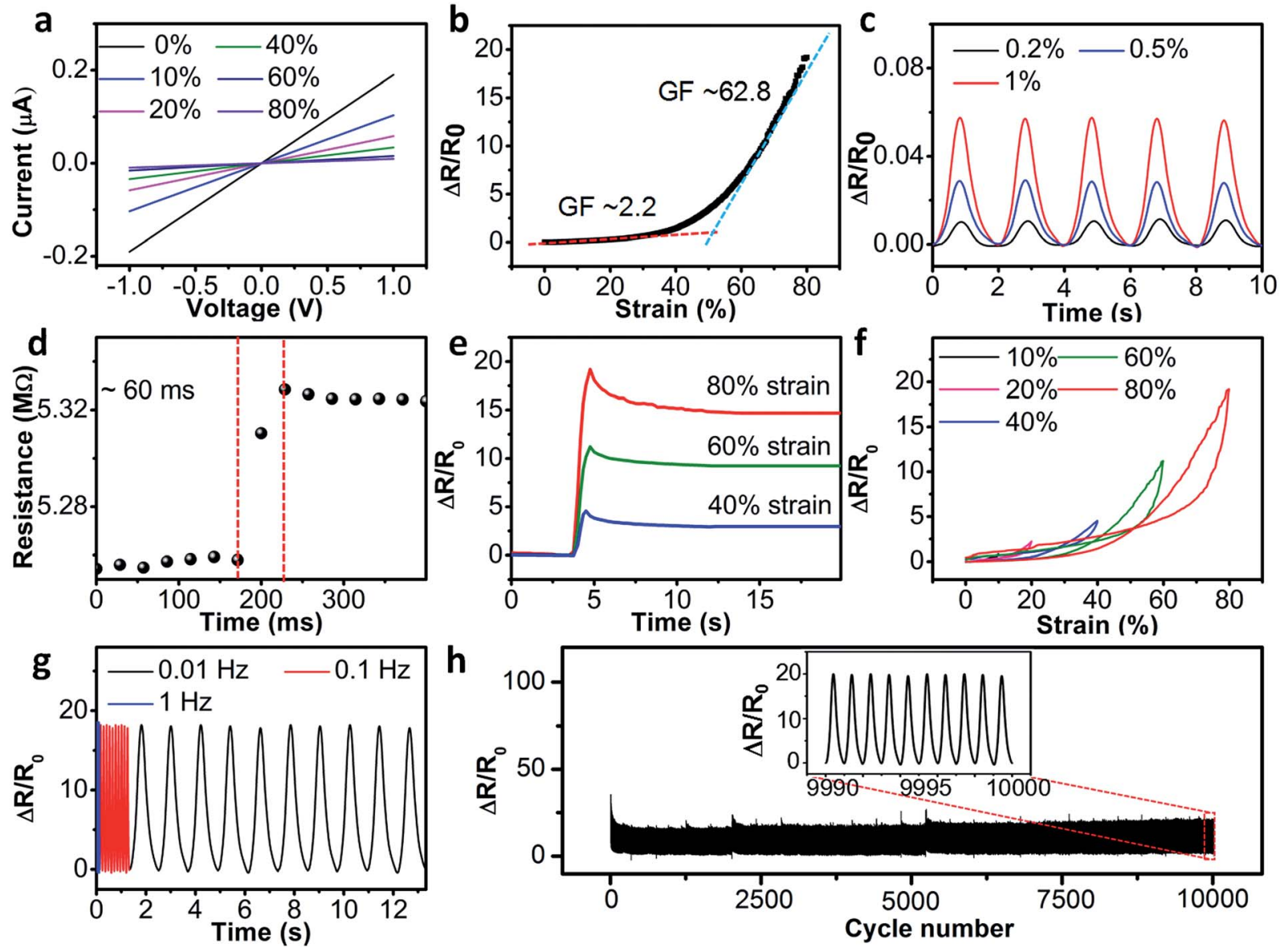

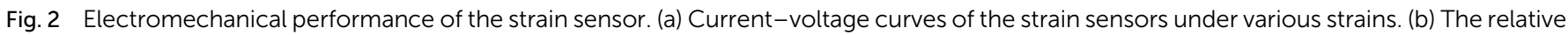

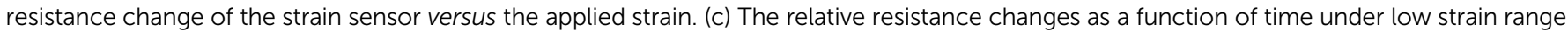

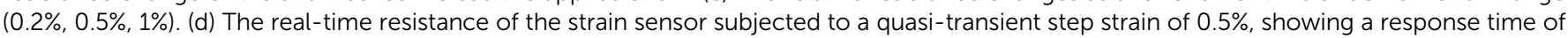

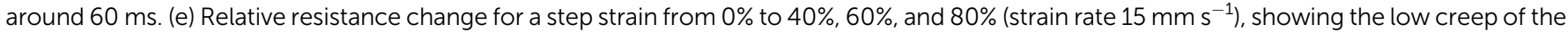

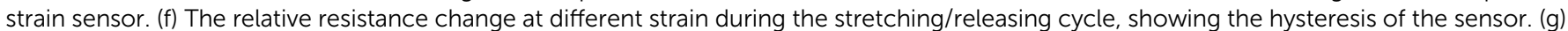

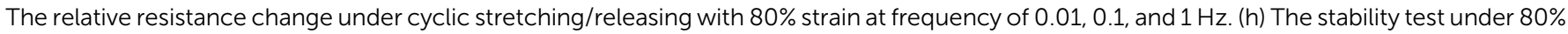
strain. 
walking, jumping and jogging. Important in health-caring application, the sensibility for monitoring small-scale movements (e.g., wrist pulse, breathing, speaking) was investigated by testing the relative resistance change upon the strain as low as $0.2 \%$. Subjected to cyclic low strains $(0.2-1 \%)$, the relative resistance change was able to be detected in a highly repeatable manner (Fig. 2c), demonstrating the stable and reliable sensing performances at low strain region.

In addition to the high sensitivity across a wide workable range, our strain sensor exhibited fast response and recovery. Fig. $2 \mathrm{~d}$ presented the response to a quasi-transient step strain of $0.5 \%$ and the responsive time was $\sim 60 \mathrm{~ms}$. The recovery time from large strains were also evaluated by stretching the sensor to $40 \%, 60 \%$ and $80 \%$ strains at a speed of $15 \mathrm{~mm} \mathrm{~s}^{-1}$ (Fig. 2e). Correspondingly, the sensor was recovered within 3,7 , and 10 seconds upon strain releasing. The narrow hysteresis loop of the relative resistance changes was observed even with the strain up to $80 \%$, which was attributed to a full-recovery of the microscopic structure of the strain sensor from large deformation (Fig. 2f). It was worth to note that fast response and recovery would be a big challenge for filler-type strain sensor because the interfacial frictions between fillers and polymer matrix would cause a big lag of signal behind applied strains. ${ }^{38}$ As described in the processing part, we paid efforts to achieve the exceptionally uniform distribution of CFs in the PDMS matrix of our sensor, in which each CF was capable to apportion the large deformation to minimize the local deformation and interfacial friction. As shown in Table $\mathrm{S} 1, \uparrow$ the responsive time of our sensor was among the best values for filler-type strain sensors, which was $\sim 10$ times faster than that of carbon black/ polymer composite (relaxation times of more than 100 seconds). ${ }^{39}$ The fast response and recovery ensured the frequency stability upon high strains up to $80 \%$, which had never been achieved by any stretchable sensors before.

To monitor diverse human body movements at different motion speed, we measured the relative resistance change of the strain sensor over the frequency range from 0.01 to $1 \mathrm{~Hz}$, which almost covered the motion speed of human's daily activities at different tensile strains. As shown in Fig. $2 \mathrm{~g}$, the relative resistance change $\left(\Delta R / R_{0}\right)$ of the strain sensor showed negligible changes over the frequency range from 0.01 to $1 \mathrm{~Hz}$ at the $80 \%$ strain. In the previous reports, the frequency stability of PDMS-based strain sensors was only achieved within the strain range less than $50 \% .{ }^{27-29}$ It provided our strain sensors with the ability to monitor the large-scale movements of human body.

The strain sensor also exhibited excellent durability at high strain levels. Fig. $2 \mathrm{~h}$ showed the tests under a cyclic strain of $0-$ $80-0 \%$ with stage moving speed at $2 \mathrm{~mm} \mathrm{~s}^{-1}$. As the Fig. $2 \mathrm{~h}$ depicted, the strain sensor responded to the cyclic loading for more than 10000 cycles with a very good stability. The relative resistance change of strain sensor showed negligible changes after the cyclic test, demonstrating the high durability of our stretchable sensors at high strains.

For a deep insight of the working principle of our sensor, the CFs was sprayed onto the PDMS film and the structural evolution of conductive pathway was investigated under both optical and microscopic observation throughout the loading-unloading cycle (Fig. 3a and b). At $0 \%$ strain, the optical images showed the continuous conductive pathways, indicated by the low light transmission. When the sample was being stretched to $80 \%$, the bright spots were gradually produced which was associated with the separation of neighboring CFs. These spots, representing CF separations, evenly distributed throughout the deformation region with the maximum size no more than 20 $\mu \mathrm{m}$. They shrank with the time till disappearing after strain releasing, indicating the recovery of the CFs to their original position and re-acquire self-contacting mode at the submacroscopic level (Fig. 3a). To further reveal the variation of CFs morphology at stretching, we conducted SEM investigation that the separation of the neighboring CFs was observed with the decrease of CF-CF contacts (Fig. 3b). Once the strain was released, the CFs bounced back to reform the conductive pathway with the help of elastic PDMS.

We confirmed that the breakage of the conductive network functioned the sensitivity of our sensor, which was similar with the strain sensors comprising the graphene flakes ${ }^{35}$ and graphene fragments. ${ }^{20}$ Also, it explained the trend of the tuning of sensing properties by changing the CF/IPA dispersion, which agreed with the tailoring rule based on derivative of the resistance-strain function. ${ }^{35}$ For a comparison, same amount of multi-layer rGO were used to prepare the strain sensor. As shown in Fig. 3c, the rGO-based conductive pathway showed inhomogeneous distribution. Upon stretching, we saw large rips happening to the conductive pathway. Different from the "tiny" separation of the CF units, the rGO network showed large part "migration" up to hundreds of micrometers, which lost the reversibility to its original structure upon strain releasing (Fig. 3b and d).

It was our understanding that one of the key aspects in our current work, compared with the previous work by using synthetic nanocarbon materials (e.g. graphene) as conductive fillers, was the appropriate control of $\mathrm{CF}$ size during the fabrication process. From a mechanical standpoint, the structural design for a highly stretchable, fast responsive and highly durable material is required to minimize geometric discontinuities, since geometric discontinuities can cause the local increase in the intensity of a stress field. It is the stress concentration we mentioned above. Examples of objects that cause the stress concentration are cracks, holes and interfaces between the matrix and filler in the example of composite materials. This is the reason why filler materials need to be dispersed as adequately as possible to prevent/reduce the stress concentration occurred at interfaces in composite materials. Particularly, during the cyclic operation at high strains, the stress concentrations prefer to be released by cracks, and would propagate and connect to cause the fatigue failure of materials.

In our case, to achieve a durable strain sensor with fast response and frequency stability at high strain levels, the CFs proceeded multi-level filtration before being mixed with PDMS matrix to minimize the geometric discontinuities as described above. According to the particle counters testing conducted after removal of PDMS, the particle size of CFs showed a highly uniform distribution with a single sharp peak at $16.4 \mu \mathrm{m}$. It not 

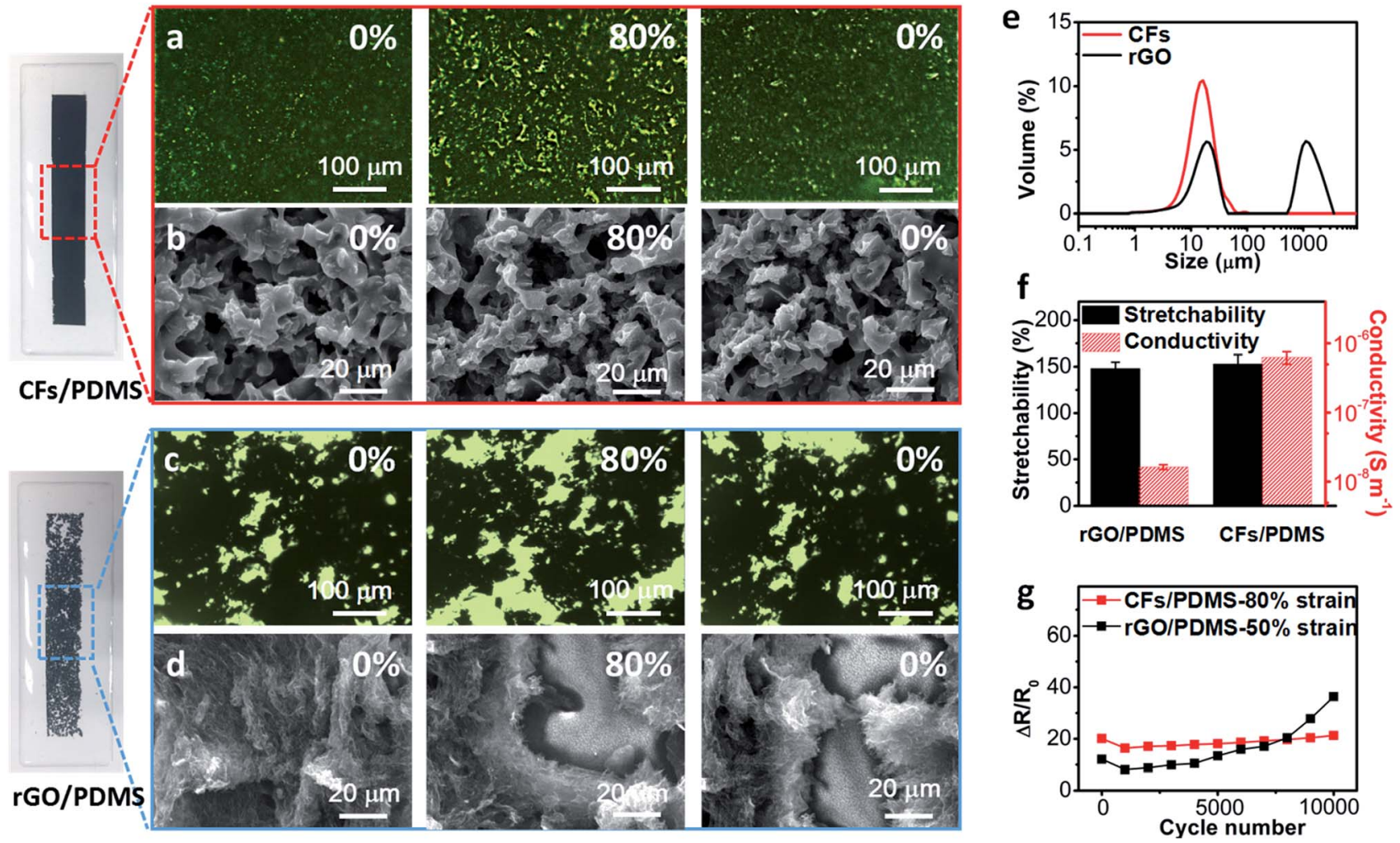

Fig. 3 Optical microscope images and SEM images of surface morphology of the (a, b) CFs/PDMS composite and (c, d) rGO/PDMS composite at different strains. (e) The particle size distribution of the CFs and rGO. (f) The maximum stretchability and electrical conductivity of the two strain sensors. (g) The cycle stability of the two strain sensors at $80 \%$ strain and $50 \%$ strain, respectively.

only lowered the stress concentration as much as possible, but also enhanced the uniformity of conductive pathways to promote the sensing stability at stretching. In contrast, rGO flakes showed two distinguished peaks located at $18.7 \mu \mathrm{m}$ and $1.11 \mathrm{~mm}$. Even under the same filtration processing and adequate dispersion, the re-aggregation of these synthetic nanomaterials still occurred during mixing process (Fig. 3e). It highlighted the advantage of using wheat bran derived carbonaceous materials over nanocarbon materials that it was capable to be transformed to highly conductive carbon materials with the aggregation inhibited either during dispersion or mixing process. Although their stretchability showed the similarity to each other, the electrical conductivity of the wheat bran derived carbonaceous materials strain sensor was $\sim 38$ times higher than that of the rGO sensor (Fig. 3f), which granted a wider operational range up to $153 \%$ than the $54 \%$ of rGO sensor (see Fig. S9†). More importantly, the wheat bran derived carbonaceous materials strain sensor exhibited the obvious improvement on the durability at high strains. As mentioned above, our wheat-sourced strain sensor behaved stably over 10000 cycles at $80 \%$ strain, which showed a highest ultimate cyclic stretching strain level among carbon-based strain sensors (see Table S1 $\dagger$ ). The ultimate cyclic stretching strain was associated with the "cracking-repairing" ability of microstructure at specific deformation level. When the applied strain exceeded $80 \%$, the bright spots were connected into strips. It meant the CF separations were turned into big cracks, which could not be repaired upon releasing (see Fig. S10†). As a comparison, the rGO sensor showed gradual increasing of resistance once exceeding 2000 cycles even under a lower strain of 50\% (Fig. 3g).

As seen, we minimized the local deformation of each CF through the fragment size controlling, which turned out to improve the microscopic "cracking-repairing" ability at high strains by minimizing CF separations efficiently. The interfacial stress concentration was expected to be reduced at utmost, thereby preventing the cracking production (Fig. 4a). For the rGO-based sensor, the cracks observed were ranged from hundreds of nanometers up to 100 micrometers due to the geometric discontinuity caused by aggregation (Fig. 4c). The big-sized cracks easily propagated during the cyclic working, exhibiting a gradual increasing of resistance as a sign. Finally they connected to form macroscopic crack until the breaking of the sensor.

All these interpretations can be supported by the microstructure evolution of our sensors examined by SEM observations (Fig. $4 \mathrm{~b}$ and d). For our wheat bran derived carbonaceous materials sensor, no visible cracking was found at $80 \%$ strain, the ultimate cyclic strain at which the "cracking-repairing" ability still being possessed. Even after 10000 stretching cycles, the structure behaved almost identically as original (Fig. $4 \mathrm{~b}, 0 \%$ strain after releasing). Differently, the rGO-based sensor showed serious cracking at 50\% strain (Fig. 4d, 50\% strain). These cracks closed up after strain releasing. Yet apparently, the length of cracks increased by the evidence of the roughened 


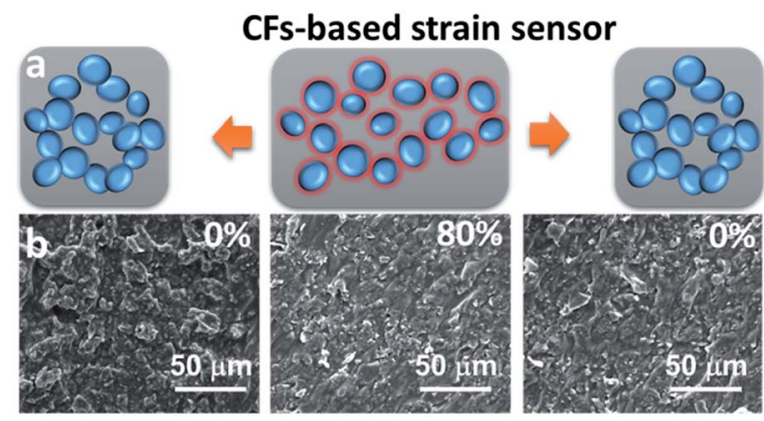

rGO-based strain sensor
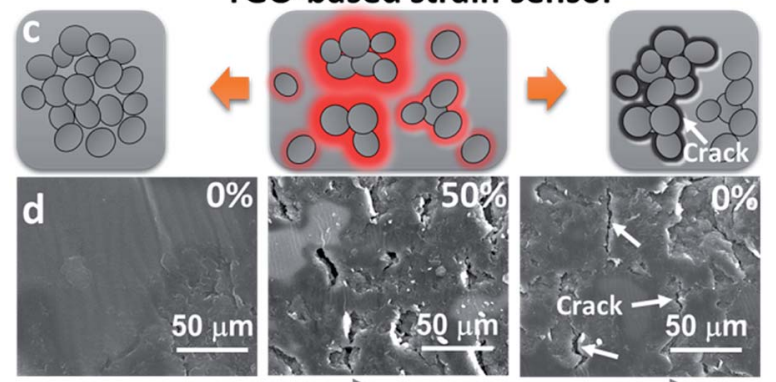

Stretching

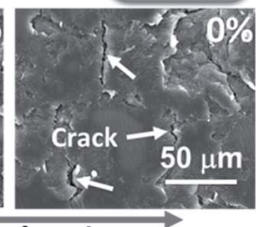

Releasing

Fig. 4 Schematic illustration of the deformation of the (a) CFs-based and (c) rGO-based strain sensor during stretching/releasing cycle. The red parts represent the interfacial stress concentration of the strain sensor. The blue and grey spheres represent the CFs and rGO, respectively. SEM images of the surface of the (b) CFs-based and (d) rGO-based strain sensor at different states.

surface compared to the one before stretching. Beneath the PDMS covering, the cracks elongated around the rGO flakes, giving rise to their peeling from matrix (Fig. $4 \mathrm{~d}, 0 \%$ strain after releasing).

The durability and high stretchability of our strain sensor made it attractive for monitoring full-range human body movements, ranging from small-scale movements (e.g., subtle
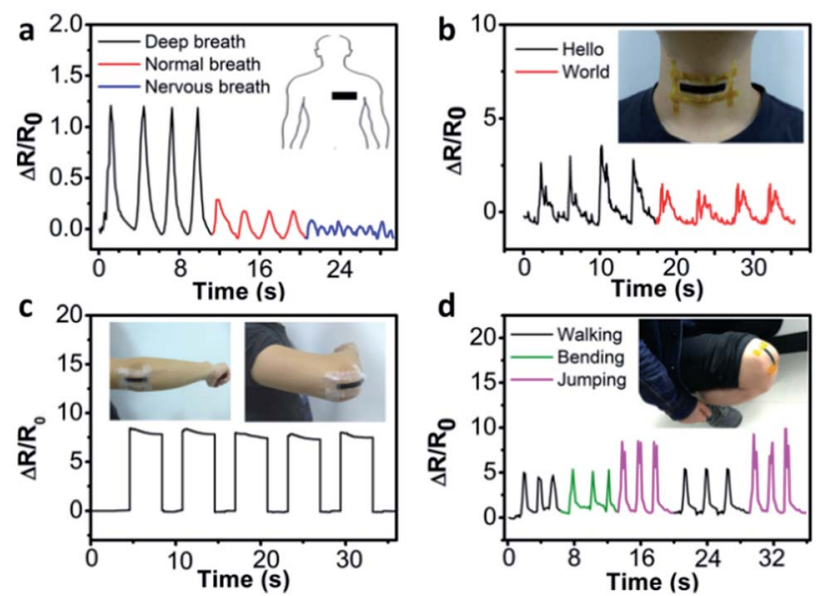

Fig. 5 Demonstration of strain sensor for human movements monitoring. The relative resistance changes of the strain sensor versus time for (a) breathing, (b) speaking, (c) elbow bending and (d) bending, walking and jumping of knee joint. movements in the wrist pulse, breathing, speaking) to largescale movements (e.g., bending movements of elbows) (Fig. 5). To detect various movements of human body, our strain sensor was attached to the human body using the tape. As vital signs, wrist pulse and breathing were important to be detected for personal health. The wrist pulse numbers were 15 within $10 \mathrm{~s}$ and the data was within the normal range of a healthy adult (see Fig. S11†). By attaching the strain sensor to the chest region of human body, the sensor was able to monitor different breathing patterns (deep, normal and nervous breathing) with continuous relative resistance changes under repeated breathing (Fig. 5a). The peaks and valleys were attributed to the chest stretch and shrink, respectively. The amplitude and frequency of different breathing patterns were successfully recorded in real-time. This property was expected to be used as monitoring device for people with sleep apnea. Also, our strain sensor was able to monitor speech by detecting the complex movements of skin of neck, as shown in Fig. 5b. To sense large-scale human body movements, we attached the strain sensor onto the elbow. The resistance change was recorded in real time during the repeated bending processes of the elbow. Fig. $5 \mathrm{c}$ showed that the resistance increased with the bending of elbow and remained unchanged when the elbow kept bending posture. In addition, we attached the strain sensor onto the knee joint to monitor different movements of knee joints, such as bending of knee joint, walking and jumping (Fig. 5d).

\section{Conclusions}

In summary, we fabricated a wearable strain sensor possessing the high stretchability and sensitivity using carbon fragments derived from wheat bran derived carbonaceous materials as conductive materials. The strain sensors showed high stretchability of $\sim 80 \%$, and GF of 2.2 (for strain of $0-40 \%$ ) and 62.8 (for strain of $40-80 \%)$. Furthermore, the strain sensor had high durability over 10000 stretching cycles at $80 \%$ strain, with the frequency stability over $0.01-1 \mathrm{~Hz}$. The key to acquire the comprehensively sensing performance was to appreciate the need for developing a composite structure with the exceptionally uniform distribution of carbon fragments in polymer matrix, which possessed the cracking-repairing ability at large strains by minimizing stress concentrations. The excellent sensing performance of the strain sensor enabled it to monitor in real-time strain signals from subtle skin stretching to largescale joint movement. The methodologies developed in this study for the materials preparation and strain sensor device construction/integration are applicable to the development of various new multi-functional sensing devices for many other applications, including robotic skins, prosthetic limbs and wearable electronics.

\section{Acknowledgements}

This work was jointly supported by the National Thousand Talents Plan of China, the National Natural Science Foundation of China (51402117, 51572095), State Key Laboratory of Materials Processing and Die \& Mould Technology, Fundamental 
Research Funds for the Central Universities, and Shenzhen Basic Research Project (JCYJ20140903171444756). The authors thank the Analytical and Testing Center of Huazhong University of Science and Technology for the assistance provided to sample characterization.

\section{References}

1 D. Son, J. Lee, S. Qiao, R. Ghaffari, J. Kim, J. E. Lee, C. Song, S. J. Kim, D. J. Lee, S. W. Jun, S. Yang, M. Park, J. Shin, K. Do, M. Lee, K. Kang, C. S. Hwang, N. Lu, T. Hyeon and D.-H. Kim, Nat. Nanotechnol., 2014, 9, 397.

2 S. Park, H. Kim, M. Vosgueritchian, S. Cheon, H. Kim, J. H. Koo, T. R. Kim, S. Lee, G. Schwartz, H. Chang and Z. Bao, Adv. Mater., 2014, 26, 7324.

3 E. Roh, B.-U. Hwang, D. Kim, B.-Y. Kim and N.-E. Lee, ACS Nano, 2015, 9, 6252.

4 Y. Wang, L. Wang, T. Yang, X. Li, X. Zang, M. Zhu, K. Wang, D. Wu and H. Zhu, Adv. Funct. Mater., 2014, 24, 4666.

5 M. L. Hammock, A. Chortos, B. C.-K. Tee, J. B.-H. Tok and Z. Bao, Adv. Mater., 2013, 25, 5997.

6 K.-Y. Chun, Y. Oh, J. Rho, J.-H. Ahn, Y.-J. Kim, H. R. Choi and S. Baik, Nat. Nanotechnol., 2010, 5, 853.

7 M. Yanilmaz, F. Kalaoglu, H. Karakas and A. S. Sarac, J. Appl. Polym. Sci., 2012, 125, 4100.

8 H. Stoyanov, M. Kollosche, S. Risse, R. Wache and G. Kofod, Adv. Mater., 2013, 25, 578.

9 S. Rosset and H. R. Shea, Appl. Phys. A: Mater. Sci. Process., 2013, 110, 281.

10 M. Amjadi, A. Pichitpajongkit, S. Lee, S. Ryu and I. Park, ACS Nano, 2014, 8, 5154.

11 S. Gong, D. T. H. Lai, B. Su, K. J. Si, Z. Ma, L. W. Yap, P. Guo and W. Cheng, Adv. Electron. Mater., 2015, 1400063.

12 M. Amjadi, Y. J. Yoom and I. Park, Nanotechnology, 2015, 26, 375501.

13 F. M. Guo, X. Cui, K. L. Wang and J. Q. Wei, Nanoscale, 2016, 8, 19352.

14 Y. Qin, Q. Peng, Y. Ding, Z. Lin, C. Wang, Y. Li, F. Xu, J. Li, Y. Yuan, Y. He and Y. Li, ACS Nano, 2015, 9, 8933.

15 C. Yan, J. Wang, W. Kang, M. Cui, X. Wang, C. Y. Foo, K. J. Chee and P. S. Lee, Adv. Mater., 2014, 26, 2022.

16 D. H. Ho, Q. Sun, S. Y. Kim, J. T. Han, D. H. Kim and J. H. Cho, Adv. Mater., 2016, 28, 2601.

17 Y. Cheng, R. Wang, J. Sun and L. Gao, Adv. Mater., 2015, 27, 7365.

18 Q. Liu, J. Chen, Y. Li and G. Shi, ACS Nano, 2016, 10, 7901.
19 C. S. Boland, U. Khan, C. Backes, A. O'Neill, J. McCauley, S. Duane, R. Shanker, Y. Liu, I. Jurewicz, A. B. Dalton and J. N. Coleman, ACS Nano, 2014, 8, 8819.

20 Y. R. Jeong, H. Park, S. W. Jin, S. Y. Hong, S.-S. Lee and J. S. Ha, Adv. Funct. Mater., 2015, 25, 4228.

21 J. Lee, S. Kim, J. Lee, D. Yang, B. C. Park, S. Ryu and I. Park, Nanoscale, 2014, 6, 11932.

22 G. Zhang, F. Wang, J. Dai and Z. Huang, Materials, 2016, 9, 92.

23 K. Poulios and C. F. Niordson, Compos. Struct., 2016, 157, 424.

24 M. Ha, S. Lim, J. Park, D.-S. Um, Y. Lee and H. Ko, Adv. Funct. Mater., 2015, 25, 2841.

25 K. Takei, T. Takahashi, J. C. Ho, H. Ko, A. G. Gillies, P. W. Leu, R. S. Fearing and A. Javey, Nat. Mater., 2010, 9, 821.

26 Y. Li, Y. A. Samad and K. Liao, J. Mater. Chem. A, 2015, 3, 2181.

27 Y. Li, Y. A. Samad, T. Taha, G. Cai, S.-Y. Fu and K. Liao, ACS Sustainable Chem. Eng., 2016, 4, 4288.

28 M. Zhang, C. Wang, H. Wang, M. Jian, X. Hao and Y. Zhang, Adv. Funct. Mater., 2017, 27, 1604795.

29 C. Wang, X. Li, E. Gao, M. Jian, K. Xia, Q. Wang, Z. Xu, T. Ren and Y. Zhang, Adv. Mater., 2016, 28, 6640.

30 D. C. Marcano, D. V. Kosynkin, J. M. Berlin, A. Sinitskii, Z. Sun, A. Slesarev, L. B. Alemany, W. Lu and J. M. Tour, ACS Nano, 2010, 4, 4806.

31 S. Stankovich, D. A. Dikin, R. D. Piner, K. A. Kohlhaas, A. Kleinhammes, Y. Jia, Y. Wu, S. T. Nguyen and R. S. Ruoff, Carbon, 2007, 45, 1558.

32 M. Wahid, G. Parte, D. Phasec and S. Ogale, J. Mater. Chem. A, 2015, 3, 1208.

33 L. Hu, J. Hou, Y. Ma, H. Li and T. Zhai, J. Mater. Chem. A, 2016, 4, 15006.

34 Z. Li, Z. Xu, X. Tan, H. Wang, C. M. B. Holt, T. Stephenson, B. C. Olsenab and D. Mitlin, Energy Environ. Sci., 2013, 6, 871.

35 M. Hempel, D. Nezich, J. Kong and M. Hofmann, Nano Lett., 2012, 12, 5714.

36 C. Lee, L. Jug and E. Meng, Appl. Phys. Lett., 2013, 102, 1835111.

37 M. Park, H. Kim and J. P. Youngblood, Nanotechnology, 2008, 19, 055705.

38 T. Yamada, Y. Hayamizu, Y. Yamamoto, Y. Yomogida, A. Izadi-Najafabadi, D. N. Futaba and K. Hata, Nat. Nanotechnol., 2011, 6, 296.

39 C. Mattmann, F. Clemens and G. Tröster, Sensors, 2008, 8, 3719. 